\title{
Position criticality in chess endgames
}

\author{
Book or Report Section
}

Accepted Version

post conference postscript

Haworth, G. and Rusz, A. (2012) Position criticality in chess endgames. In: Advances in Computer Games. Lecture Notes in Computer Science, 7168. Springer, Heidelberg, pp. 244257. doi: https://doi.org/10.1007/978-3-642-31866-5_21 Available at http://centaur.reading.ac.uk/23799/

It is advisable to refer to the publisher's version if you intend to cite from the work. See Guidance on citing.

To link to this article DOI: http://dx.doi.org/10.1007/978-3-642-31866-5_21

Publisher: Springer

All outputs in CentAUR are protected by Intellectual Property Rights law, including copyright law. Copyright and IPR is retained by the creators or other copyright holders. Terms and conditions for use of this material are defined in the End User Agreement.

www.reading.ac.uk/centaur 


\section{CentAUR}

Central Archive at the University of Reading

Reading's research outputs online 


\title{
Position Criticality in Chess Endgames
}

\author{
G.M $^{\mathrm{c}}$ C.Haworth ${ }^{1}$ and Á. Rusz ${ }^{2}$ \\ ${ }^{1}$ School of Systems Engineering, University of Reading, \\ guy.haworth@bnc.oxon.org \\ ${ }^{2}$ Budapest, Hungary
}

\begin{abstract}
Some 50,000 Win Studies in Chess challenge White to find an effectively unique route to a win. Judging the impact of less than absolute uniqueness requires both technical analysis and artistic judgment. Here, for the first time, an algorithm is defined to help analyse uniqueness in endgame positions objectively. The key idea is to examine how critical certain positions are to White in achieving the win. The algorithm uses sub- $n$-man endgame tables (EGTs) for both Chess and relevant, adjacent variants of Chess. It challenges authors of EGT generators to generalise them to create EGTs for these chess variants. It has already proved efficient and effective in an implementation for Starchess, itself a variant of chess. The approach also addresses a number of similar questions arising in endgame theory, games and compositions.
\end{abstract}

Keywords: chess variant, endgame, repetition, Starchess, study, uniqueness

\section{Introduction}

A Win Study in Chess is a composition in which White is challenged to win against Black's best defence. White's choice of move at each stage should be effectively unique even if not absolutely unique as in Sudoku or a crossword. Where there is more than one goal-compatible move, questions arise about the technical integrity and artistic quality of the study. The incidence of sub-7-man (s7m) mainline DTM-equioptimal and DTM-sub-optimal moves in the HHDBIV corpus of over 76,000 studies [1] has been profiled [2] using Nalimov EGTs [3-4]. The comments of leading solvers, editors and judges of studies make it clear that the effective uniqueness question is arguably the Grand Challenge for the study community. Beasley: "the detection of blind alleys in general is notoriously difficult." Roycroft: "When the depth difference is greater than two or three, one tends to shrug and move on to something else." Nunn: "detecting cycling moves can be easy in the case of a simple repetition or can be essentially impossible to do by hand in very complex cases."

Chess is a second-generation variant of a germinal board game and has inspired its own large family of variants [5-6]. It is unfortunately still necessary to note that the Nalimov EGTs are still in fact for a variant of chess without castling. ${ }^{1}$ The primary goal of chess variants is to provide an entertaining new challenge but the less radical

\footnotetext{
${ }^{1}$ Creating supplementary EGTs for positions with castling rights is in fact a small task.
} 
variants also inform about chess itself. The creation of EGTs with restrictions on underpromotions [7-10] has, when compared with the standard EGTs, revealed spectacular and essential underpromotions: see the positions $U P i$ in Table 1, Figure 1 and the Appendix. The impact of the 50-move draw-claim rule has been noted [11] after computing EGTs for the chess variant Chess $_{50}$ in which phases with more than 50 winner's moves were deemed drawn. ${ }^{2}$ Looking at the effect of giving the defender the null move has been proposed [12] and similarly, one might consider the effect of removing the ability either to capture or to mate in the current phase of play. ${ }^{3}$

The proposal here is to address the study community's effective uniqueness question algorithmically using EGTs. This is to be done by defining appropriate chess variants Chess $(S P): S P$ is a set of positions, each won for White in Chess but defined to be a draw in Chess(SP). The impact of these changes on the values of positions in another set $T P$ of White wins is a measure of the criticality or importance of the positions in $S P$ to those in $T P$. It may be determined from the difference, $\Delta\left(E G T, E G T_{S P}\right)$, between Chess' EGT and Chess(SP)'s EGT.

Section 2 defines a set of scenarios where the questions about position criticality may be addressed using the Chess(SP) approach: section 3 defines the response to each scenario. Section 4 details the algorithm, considers available efficiencies and estimates the workload in the context of Chess' Win Studies [1]. Section 5 reviews the first implementation and production use of the approach by the second author in the game of Starchess.

Table 1. The cited exemplar positions

\begin{tabular}{|c|c|c|c|c|c|c|c|c|}
\hline Id & Date & HHdbIV & Force & Position & move & Val. & DTM & Notes \\
\hline UP1 & 2000 & --- & KQQKQP & wKg4,Qg8,h8/bKf1,Qb4,Pd2 & $\mathrm{w}$ & $1-0$ & 60 & Karrer: $\mathrm{DTM}=20$ if $\mathrm{dl}=\mathrm{N}$ is not possible \\
\hline UP2 & 2009 & 75917 & KQPKQ & wKc8,Qf4,Pg7/bKh5,Qh1 & $\mathrm{w}$ & $1-0$ & 13 & Konoval and Bourzutschky: $\mathrm{P}=\mathrm{R}$ and $=\mathrm{N}$ \\
\hline UP3 & 2010 & --. & KRRPKQ & $\mathrm{wKc} 2, \operatorname{Rb} 5, \mathrm{~d} 5, \operatorname{Pg} 5 / \mathrm{bKh} 5, \mathrm{Qf} 8$ & $\mathrm{w}$ & $1-0$ & 36 & Konoval and Bourzutschky: $\mathrm{P}=\mathrm{R},=\mathrm{B}$ and $=\mathrm{N}$ \\
\hline $\mathrm{S} 1$ & 1895 & 3477 & KPKR & wKb6,Pc6/bKa1,Rd5 & w 1 & $1-0$ & 26 & Saavedra and Barbier: most documented study \\
\hline S2 & 2009 & 75649 & КРPРКРPP & wKfl,Pa4,d5,g5/bKh8,Pa5,d6,g7 & w 1 & $1-0$ & 83 & Hornecker study \\
\hline S2' & 2009 & 75649 & KQPKQP & wKc6,Qf1,Pd5/bKg5,Qd4,Pd6 & w 17 & $1-0$ & 64 & Hornecker study: sideline after 16. Qf1+ \\
\hline S3 & 1924 & 9797 & KRI & wKc6,Ne5,Rg5/bKd8,Nf8,h6 & w 1 & $1-0$ & 26 & hing moves at pos. $1 \mathrm{w}$ \\
\hline S4 & 1924 & 9686 & KNPKPP & wKf1,Ne2,Pg2/bKe3,Pf4,g3 & $\mathrm{w}$ & $1-0$ & 36 & Reti and Mandler study; $\mathrm{p} 3 \mathrm{w}$ is a B1-M zug \\
\hline $\mathrm{PH}$ & 1777 & 956 & KQKR & wKc6,Qa5/bKb8,Rb7 & $\mathrm{w}$ & $1-0$ & 10 & Philidor: B1 zug \\
\hline $\mathrm{KH}$ & 1851 & 1822 & BBKN & $\mathrm{wKd} 5, \mathrm{Ba} 4, \mathrm{f8} / \mathrm{bKb} 6, \mathrm{Nb} 7$ & $\mathrm{w}$ & $1-0$ & 57 & Pseudo-fortress long thought to be drawn \\
\hline B1Z & 2011 & -- & КРPKРP & wKg5,Pe6,f7/bKg7,Pe7,g6 & $\mathrm{w}$ & $1-0$ & 18 & Elkies: a vital $\mathrm{B} 1$ zug needing $\mathrm{B} 1 \mathrm{Z} / \mathrm{btm}$ to win \\
\hline $\mathrm{B} 1 \mathrm{Z}^{\prime}$ & ' 2011 & --- & КРPKP & wKg5,Pe6,f7/bKg7,Pe7 & $\mathrm{w}$ & $1-0$ & 13 & Elkies: not a vital B1 zug \\
\hline
\end{tabular}
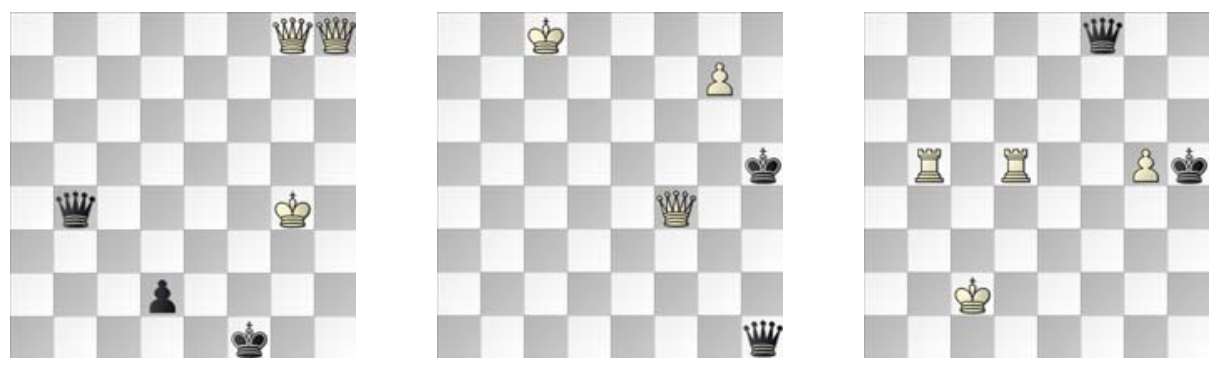

Fig. 1. Wins UP1-3 requiring underpromotions, found using Chess variant EGTs.

${ }^{2} 50.15 \%$ of wtm and $70.98 \%$ of btm wins in KBBKNN are '50-move draws' [11]. 


\section{Scenarios and questions to be considered}

The scenarios are constrained to that part of Chess for which the perfect information of EGTs is available. As is the convention in Chess Win Studies, White has a win throughout the line $\left\{P_{1}, \ldots, P_{n}\right\}$ and plays move $m_{c}: P_{c} \rightarrow P_{c+1}$ from the 'current' position. However, there may be alternative value-preserving moves $m_{c, j}: P_{c} \rightarrow P_{c, j}$. The moves which are suboptimal in any available metric ${ }^{4}$ are $m_{c, 1}$ to $m_{c, j l}$ while those optimal in some available metric are $m_{c, j l+1}$ to $m_{c, j 2}$. SP and TP are two sets of positions theoretically won for White. Chess $(S P)$ is a variant of chess only in that the positions in $S P$ are deemed to be drawn, perhaps creating further draws in Chess(SP). ${ }^{5}$ The question is 'What are TP's positions' values in Chess(SP)?' This notation is used:

$S_{i} \equiv$ a reference to Table $1,{ }^{!} \equiv$ a move which seems clearly the best,

$\rightarrow \equiv$ a move, $p n(w / b) \equiv(\mathrm{wtm} / \mathrm{btm})$ as at position $n, t w \equiv$ time-wasting move,

' and " $\equiv$ DT $x$-optimal, DT $x$ being DTM here,

(1) $\equiv$ all DT $x$-suboptimal moves are time-wasting moves,

"' $\equiv$ the unique value-preserving move, ${ }^{\circ} \equiv$ the only move available,

$(v) z \equiv$ (vital) zugzwang, $( \pm n) \equiv$ a concession of $n$ moves in DT $x$ terms,

$\mathrm{O} \equiv$ White to move (wtm) position, $\bigcirc$ Black to move (btm) position.

\subsection{The main scenario: the Win Study}

In the main scenario, a Win Study challenges White to win. At position $P_{c}$, White plays move $m_{c}$ but the dual winning moves $m_{c, j}: P_{c} \rightarrow P_{c, j}$ are also available. The study community's Grand Challenge question then, as discussed, is 'to what extent is move $m_{c}$ unique: how significant are the dual moves?' Metric suboptimal moves $m_{c, j}, j=1, j 1$ which allow Black to force White's win either to return to one of $P_{1}-P_{c}$ or to arrive at $P_{c+1}$ more slowly are time wasters and clearly inferior to a move from $P_{c}$ which actually makes progress. The technical challenge addressed here is to discover which moves can be classified as time wasters, as a prelude to re-evaluating the essential uniqueness of the move $m_{c}$. HHDBIV has some 70,000 such $\mathrm{s} 7 \mathrm{~m}$ scenarios.

In many cases, White can switchback, retracting and repeating its last move, thereby wasting four plies. Move $m_{c, j}$ may simply be to some previous mainline position $P_{i}, i<c$, or to a position one Black move off the played line. However, $m_{c, j}$ can be the start of a large tree of alternative lines leaving the reader asking whether all options have been considered, given that neither side has to play metric-optimally. Larger move trees are less comprehensible, less easily verified, and may even fall short of a complete proof that a move is merely wasting time.

Some examples show that a generally-applicable method is required to address all situations and, regardless of their complexity, to produce uniformly and easily comprehensible, verifiable proofs about time-wasting moves. Studies $S 1-S 4$, see Figures $2-5$, will suffice to indicate the main issues and open-ended range of complexity.

\footnotetext{
3 i.e., before the next Pawn-push, capture and/or mate, when the move-count is zeroed.

${ }^{4}$ e.g., DTC $\equiv$ Depth to Conversion (of force), DTZ $\equiv$ Depth to (move-count) Zeroing move.

${ }^{5}$ As is easily proved, existing draws or Black wins in Chess remain so in Chess(SP).
} 

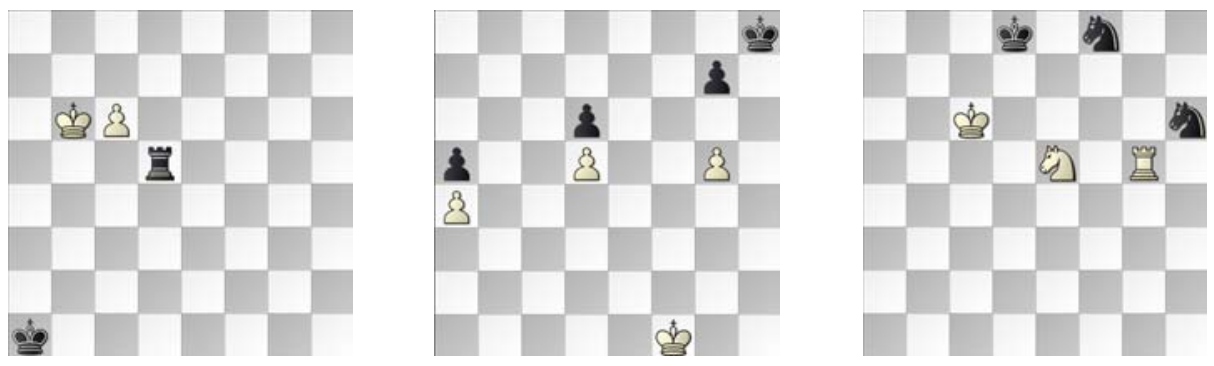

Fig. 2. Studies with ignorable time-wasting moves:

$S 1$ (Saavedra and Barbier), S2 (Hornecker) and S3 (Rinck).

The 1895 study $S 1$ by Saavedra and Barbier is justly famous for its underpromotion and colourful history [13]: its economy and brevity are also laudable. The solution is 1. c7"' $p 1 b \mathbf{R d 6 + ' ~ 2 . ~ K b 5 " ' ~ R d 5 + " ~} p 3 w$ 3. Kb4" ${ }^{(\prime)} \mathbf{R d 4 + ~ 4 . ~ K b 3 ' ~} p 4 b$ Rd3+" 5. Kc2"' Rd4! inviting the instinctive 6. c8=Q which only draws after 6. ... Rc4+"' 7. Qxc4"' stalemate. 6. c8=R" ignores White's seductive Queen by the board. After 6. ... Ra4', 7. Kb3"' wins by threatening both Rook and King.

However, White has alternative wins, see Figure 3, at moves 3, 4 and 6 which potentially undermine the uniqueness of the solution. $3 . \mathrm{Kb} 6$ and $6 . \mathrm{Kb} 3$ regress immediately to respectively $p 1 b$ and $p 4 b .4$. Kb5 allows $4 . \ldots \mathrm{Rd} 5+" p 3 w$. The increased depth of win shows that two moves have been wasted in each case. The timewasting is easy to see as the cycle is completed with at most one line and one sideline move: the solution [1] does not even acknowledge these moves. If clearly inferior moves invalidated studies on technicalities, much would be lost and the delights of such as the study by Saavedra and Barbier would be denied to a potential audience.

White also has the dual 4. Kc3' Rd1" 5.Kc2"' Rd4 p6w so it is clear that Black can force White back to the mainline downstream. A further question then about dual moves is whether they allow Black to force White's win back to the mainline and how quickly this can be done.

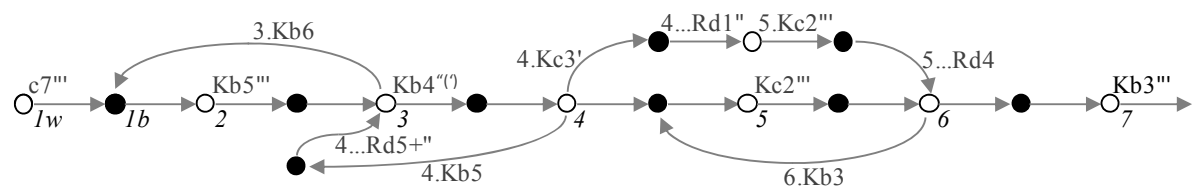

Fig. 3. A graph of the Saavedra study.

Rusz [14] recently described his more challenging demonstration of a time-wasting move which rescued Hornecker's 2009 study $S 2$. The solution is $\mathbf{1}^{\text {. g6 }}{ }^{!} \mathbf{K g 8}^{\circ} \mathbf{2 . ~ K e 2}^{!}$ $\mathrm{Kf8}^{!}$3. Kd3! Ke7! 4. Kc4! Kf6! 5. Kb5! Kxg6! 6. Kxa5! $\{$KPPKPP, DTM $=-77\}$ Kf5" 7. Kb5"' g5" 8. a5"' g4" 9. a6"' g3" 10. a7"' g2" 11. a8=Q"' g1=Q" 12. Qa3"' Ke4" 13. Kc6"' Qd4" $p 14 w$ 14. Qa5"' Kf5" $p 15 w$ 15. Qb5"(') (15. Qa3 tw Ke4" 16. Qa5 "' p14b) 15. ... Kf6 p16w 16. Qb6"(') p16b Qxb6+ 17. Kxb6"' Kf5' 18.Kc7"' Ke5' 19. Kc6"', a type A3 Trébuchet zugzwang or zug lost for Black. 


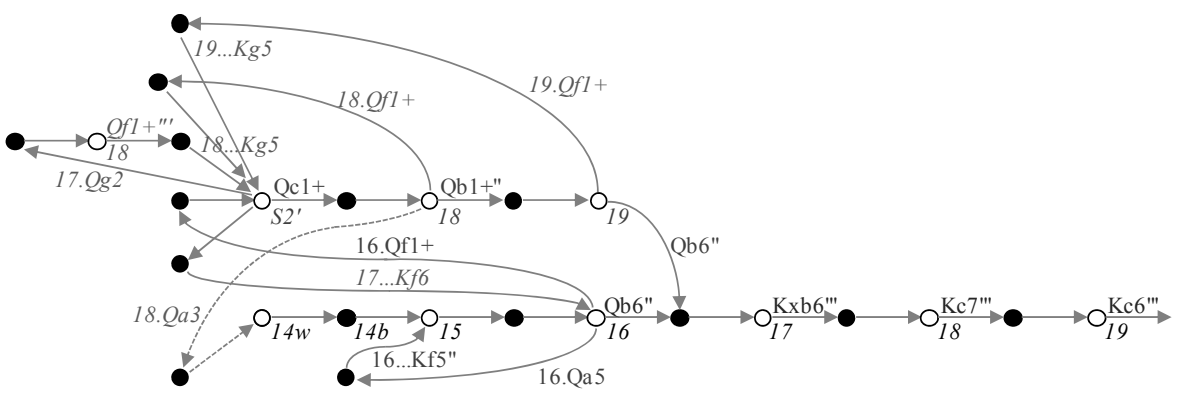

Fig. 4. A graph of the Hornecker study from position $p 14 w$.

Position pl6w is the focus: here, 20 sideline moves are required to show that White's moves 16. Qa5 and 16.Qf1+ are no more than time-wasters. 16. Qf1+ is ultimately shown to progress no further than the mainline move 16.Qb6"(1).

16. Qa5 another example of a switchback, move-reversing move 16...Kf5" $p 15 w$.

16. Qf1+ Kg5 q.v. S2' 17. Qc1+

(17.Qb5" reversing move 16w 17...Kf6 p16w; 17.Qg2+ Kf6" 18.Qf1+"' Kg5 S2')

17...Kf5 18. Qb1+"

(18.Qf1+Kg5 S2'; 18.Qa3 Ke4" p14w) 18...Kf6 19.Qb6" p16b (19.Qf1+Kg5 S2').

Although White has had no more than three winning options at any time, it is becoming clear that chess annotation and graphs reflect rather than reduce the complexity of proofs that moves are mere time-wasters. They redundantly detail White's unavailing attempts to make alternative progress rather than just stating that this is impossible. Further, manual proofs may not be complete and correct.
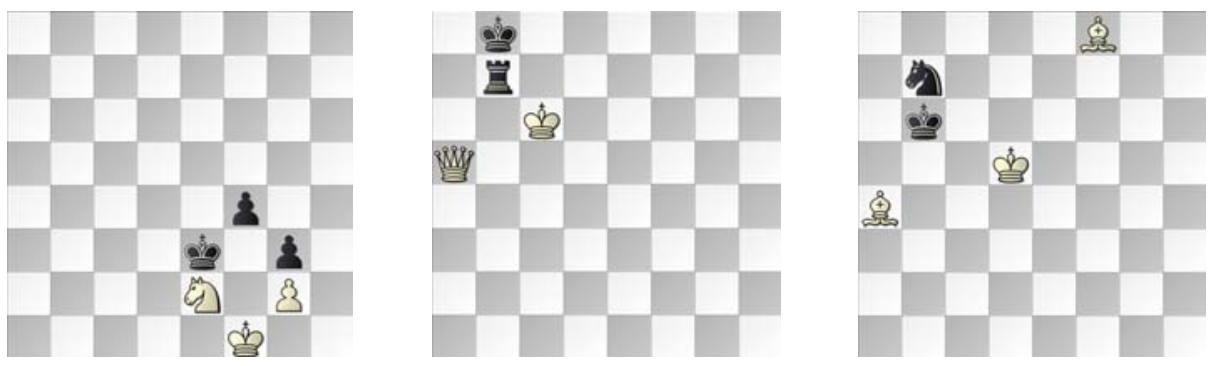

Fig. 5. $S 4$ (Reti and Mandler), $P H$ (Philidor) and $K H$ (Kling \& Horwitz).

The Rinck KRNKNN study S3 of 1924 presents an even greater challenge which is not accepted here. Rinck's solution is 1. Rh5" Ng8" 2. Rh8" Ne7+" 3. Kb5" Ke8" 4. Kc5" Nf5"/Nc8 5. Ng6" Kf7!" 6. Nxf8"' Kg7 7. Rh5/Rh1'. However 1. Rg7, $\mathrm{Kd} 5, \mathrm{Rg} 1, \mathrm{Rg} 2, \mathrm{Nc} 4$ and $\mathrm{Rg} 3$ also win. ${ }^{6}$ It is clear that these moves are not what Rinck had in mind but unclear which of them if any allow Black to force a return to the initial position. White has alternatives for all moves except the key 6. Nxf8"'.

${ }^{6}$ Conceding 30, 39, 88, 101, 132 and 170 moves in DTM terms, and 32, 41, 90, 103, 133 and 170 moves in DTC/DTZ terms. 
It is equally impractical to show via explored lines that the alternative moves in the 1924 study $S 4$ by Reti and Mandler [15] are time-wasters. The solution begins 1. Ng1"' Kd2" 2. Nf3+" Kd3", a type B1-M zug [12] [16], that is, a won position that would be won more quickly in DTM terms if White could pass. White aims to return to this physical position but with Black to move. The solution continues 3. Ke1" Ke3" 4. Ne5" Ke4" 5. Nc4" Kd3" 6. Nd2" Ke3" 7. Nf3" Kd3" 8. Kf1". At position $p 2 w, 2$. Ne2 is an obvious time-waster $(2 . . . \mathrm{Ke} 3 " p 1 w)$ but 2 . Nh3 is less easily discounted. The initial depth-concession is again only two moves but the Knight may explore the board further; similar opportunities are on offer down the main line. The analysis of these explorations in the study solution would scarcely be enlightening and the proof of time-wasting has to be in higher-order chessic terms. It would be better to use mathematical rather than chessic logic to show that 2 . Nh3 is merely a time- wasting move. White wins after 8. ... Ke3" 9. Ne1" Kd2" 10. Nc2" Kd1 11. Nb4" Kd2" 12. Nd5".

\subsection{Supplementary scenarios}

In scenario 2, White is to move from a type B1- $x$ zug. Three examples are positions $B 1 Z, B 1 Z^{\prime}$ and the Reti-Mandler study's $p 3 w$ as noted above. This suggests this question 'Is the B1- $x$ zug a vital B1 (VB1) zug, that is one from which White's win can be forced to include the btm side of the zug?' There are many more B1-M zugs in chess than value-critical zugs, and over 6,000 B1-M zugs in HHDBIV [12].

Scenario 3 sees White, a computer program, winning a game but seeking to avoid a 50-move draw-claim [17, Article 9.3] from a fallible opponent as DTR > 50 [18]. ${ }^{7}$ White's strategy is to avoid repetition ${ }^{8}[17$, Article $5.2 \mathrm{~d}]$ by considering past positions to be 'drawn', temporize and hope the opponent reduces DTR.

Scenario 4 focuses on positions highlighted as significant in endgame theory. Examples, see Figure 5, include Philidor's KQKR position $P H$ [20, Ch.3] and the Kling and Horwitz pseudo-fortress position $K H$ [19, Ch.5]. The question here is 'What related positions are not wins if these positions are not wins for White?'

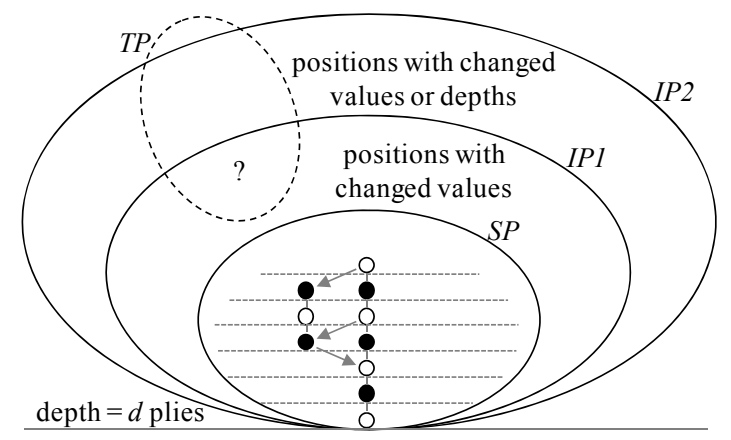

Fig. 6. Sets $I P 1$ and $I P 2$ show the impact of set $S P$, particularly on $T P$ 's positions.

${ }^{7}$ DTR $\equiv$ Depth by the (draw-claim) rule: DTR $\equiv$ smallest $k$ giving a win under a $k$-move-rule.

${ }^{8}$ Historic Dominguez-Perez/Polgar (World Cup, 2011): the unnoticed $p 95 b=p 105 b=p 107 b$ ! Rusz asks if J. Polgar can win without revisiting $p 105 b$ after regressing with $105 \ldots$ Bf5. 


\section{The algorithm: a generic response to the scenarios}

The thematic question of the scenarios is 'Given that the set $S P$ of White wins are defined to be draws, which of the White wins in set $T P$ become draws?' Figure 6 shows the set $S P, T P$ and the 'upstream' sets $I P 1$ and $I P 2$ of positions whose theoretical values or DT $x$ depths are different in Chess(SP).

The generic algorithmic response then is to:

- define the set $S P$ of White-win positions, and thus the variant game Chess $(S P)$,

- define the set TP of White-win positions whose Chess(SP) values are sought,

- compute the relevant $E G T_{S P}$ Chess(SP) EGT until set $T P$ is accounted for,

Chess(SP) like Chess has a lattice of endgame phases;

therefore, its EGTs are computable as are those of Chess,

- $\quad$ examine, for positions in set $T P$, the EGT-difference $\Delta\left(E G T, E G T_{S P}\right)$

the differences are caused only by $S P$ 's positions being draws not wins,

$P \in T P \cap I P 1 \Leftrightarrow P \in T P$ is a win in Chess but a draw in Chess(SP).

In more efficient codes, this may be seen during the computation.

This allows the computation to be aborted without generating $E G T_{S P}$ fully.

In scenario 1 at position $P_{c}, S P$ is a combination of $\left\{P_{1}, \ldots, P_{c-1}\right\},\left\{P_{c}\right\}$ and $\left\{P_{c+1}\right\}$; $T P \equiv\left\{P_{c, j}\right\}$, the set of $P_{c}$ 's successor positions, other than $P_{c+l}$, won for White. If and only if $P_{c, j}$ is in set $I P 1$, i.e., a Chess(SP) draw, the win in Chess from $P_{i, j}$ can be forced to pass through a position in $S P$. This means move $m_{c, j}$ is a time-waster, the precise reason being determined by the Chess(SP) drawing line(s) from $P_{c, j}$.

In scenario 2, $P w$ is a wtm type B1- $x$ zug and $P b$ is its btm equivalent; $S P \equiv\{P b\}$ and $T P \equiv\{P w\} . P b$ is essential to White's win from $P w$ in Chess if and only if $P w$ is a draw in Chess(SP).

In scenario 3, White has a win, may play suboptimally with regard to all metrics, but does not wish to repeat position. $S P \equiv\left\{P_{1}, \ldots, P_{c}\right\}$. Time-wasting moves to positions in $I P 1$ are avoided as are overlong phase continuations.

In scenario $4, S P \equiv\{$ significant position $\}$, e.g. position $P H$ or $K H$, is considered a near-refuge for Black. By making it an actual drawing sanctuary in Chess(SP), it is possible to assess its importance to White's winning chances in Chess.

Other scenarios involve deep wins or downstream-convergence in Win Studies, Draw Studies and value-critical zugzwangs. For clarity, these are not included in this first exposition of the Chess(SP) approach.

Table 2. HHDBIV Endgame study s7m mainline positions to be evaluated ${ }^{9}$

\begin{tabular}{ccccc}
\hline & wtm positions with alternative moves & \multicolumn{2}{c}{ EGT creation at 'Konoval tempo' (hrs) } \\
$\boldsymbol{n}$ & \#pos, $\boldsymbol{n}$ men & \#pos., $\boldsymbol{n}$ pieces & DTC/M EGTs & DTZ EGTs \\
$\mathbf{2}$ & 0 & 5,057 & $0.00 \mathrm{E}+00$ & $3.22 \mathrm{E}-03$ \\
$\mathbf{3}$ & 299 & 9,911 & $1.22 \mathrm{E}-02$ & $4.03 \mathrm{E}-01$ \\
$\mathbf{4}$ & 5,868 & 28,890 & $1.53 \mathrm{E}+01$ & $7.52 \mathrm{E}+01$ \\
$\mathbf{5}$ & 34,401 & 21,864 & $5.73 \mathrm{E}+03$ & $3.64 \mathrm{E}+03$ \\
$\mathbf{6}$ & 30,231 & 5,077 & $3.22 \mathrm{E}+05$ & $5.42 \mathrm{E}+04$ \\
Totals & 70,799 & 70,799 & $3.28 \mathrm{E}+05$ & $5.79 \mathrm{E}+04$ \\
\hline
\end{tabular}

${ }^{9}$ Chess men are pieces or Pawns: the pawns need not be moved in creating the $\mathrm{EGT}_{\mathrm{SP}}$. 


\section{Generating Chess(SP) EGTs: examples and efficiencies}

Figure 3 and a 'manual' implementation of the Chess(SP) algorithm for the study S1, position $3 w$, indicate the rapidity of the 'Chess(SP) test'. The question is whether move 3 . Kb6 is a time-waster or not. $S P \equiv\{$ pos. $3 w\}$ and $T P \equiv\{$ pos. $1 b\}$. Mainline positions $2 b, 2 w$ and $1 b$ immediately revert to draw: Black takes any drawing option and White is denied its only winning move. However, position $1 b$ was in the mainline of the study anyway so the generation of $E G T_{S P}$ was unnecessary. Showing that 4. $\mathrm{Kb} 5$ is a time-waster requires the creation of the full $E G T_{S P}$ if $S P \equiv\{$ pos. $4 w\}$. However, this is rapidly obvious if $S P \equiv$ positions $1 w-3 b$ \} when ' $3 b$ draw' implies the position after 4 . Kb5 is a draw. The reader may care to show by similar means that 6 . $\mathrm{Kb} 3$ is a time-waster and that the line starting 4 . Kc3 can be forced to position $6 w$.

If thousands of $E G T_{S P}$ designer-EGTs are to be created, it is appropriate to consider the work involved and how it might be reduced. This will depend on which EGTgenerator is evolved to create EGTs for Chess(SP). For example, Nalimov's code [3] is slower than Konoval's single-threading code which computed the KQBNKQB EGT in 3.5 weeks [21] and can compute the KQPKQ EGT in 10 minutes.

There are efficiencies which apply to creating any EGT and efficiencies which are specific to generating EGTs for Chess(SP). Konoval has used PENTIUM Assembler in the inner loops of his program and a relatively simple position-indexing scheme which facilitates the fully retrograde production of EGTs. ${ }^{10}$

EGT generation may be speeded up considerably if a trusted EGT for the same endgame is already available, especially if this is a WDL EGT. ${ }^{11}$ It is unnecessary to evaluate a position expensively as a potential loss if it is already known to be a draw or a win. This economy is in principle available when creating $\mathrm{EGT}_{\mathrm{SP}}$.

When generating $\mathrm{EGT}_{\mathrm{SP}}$, it is worth noting that:

- Chess draws and Black wins are unaffected by deeming $S P$ 's positions drawn,

- 'downstream' $Q \notin S P$ with $\mathrm{DT} x(Q) \leq \operatorname{minDT} x(P \in S P)$ are also unaffected,

- $\quad$ the $\mathrm{EGT}_{\mathrm{SP}}$ need only be for positions with Pawns in relevant positions,

- a WDL EGT $\mathrm{SP}_{\mathrm{SP}}$ is sufficient to determine set $I P 1$,

- the creation of EGT $_{\mathrm{SP}}$ may be halted when set IPI has clearly been identified or the Chess(SP) values of the positions in set $T P$ are known,

- $\quad$ iterative steps in creating $E G T_{S P}$ can identify draws as well as wins,

- a larger $S P$ does not slow the evaluation of $T P$ 's positions in Chess(SP),

- $\quad$ if $S P 2 \supset S P 1$, e.g. $\left\{P_{1} \ldots P_{c}\right\}$ and $\left\{P_{c}\right\}, \mathrm{EGT}_{\mathrm{SP} 2}$ may be derived from $\mathrm{EGT}_{\mathrm{SP} 1}$.

Based on 'Konoval performance', Table 2 estimates the computer time needed to systematically identify all time-wasting moves from the $\mathrm{s} 7 \mathrm{~m}$ mainline positions in HHDBIV's studies. The estimates do not include any Chess(SP)-specific efficiencies even though 50-fold efficiencies have been seen in Rusz' production work below. Given a suitable infrastructure to manage thousands of independent tasks, the elapsed time may be greatly reduced by the use of multi-core computers, networks of computers and crowd-sourcing. Parallelism is also possible within the set-manipulating EGTgeneration algorithm for the largest EGTs for Chess(SP).

\footnotetext{
${ }^{10}$ i.e., unmoving from positions of depth $d$ plies to discover positions of depth $d+1$ plies.

${ }^{11}$ WDL EGTs merely hold 2-bit information about wins, draws and losses but not about depths.
} 


\section{The first implementation of the algorithm: Starchess}
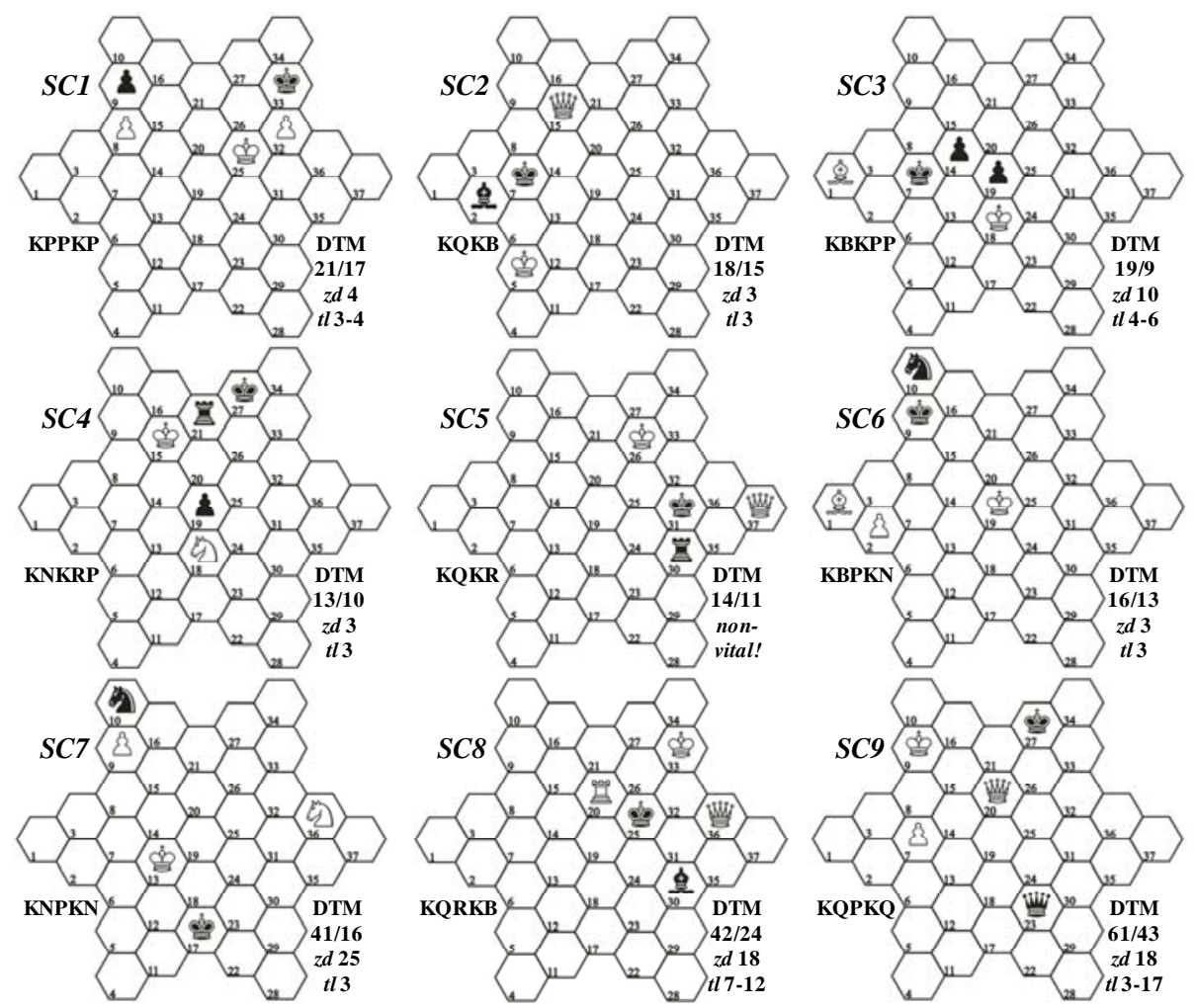

Figure 7. Sub-6-man Starchess positions SC1-9 on the Vital B1 zug theme.

As this proposal, to generate EGTs for the chess variant Chess $(S P)$, has not been widely promulgated, no existing generator of Chess EGTs has yet been generalized to do so. However, the second author, a leading authority and world champion in the game Starchess [22], has generalized his EGT generator. Starchess was invented by László Polgár in 2002 and it is only necessary here to mention the star-shaped board of just 37 hexagons ${ }^{12}$, the Knight's move, e.g., 19-2/3/9/16, and the humbled Rook which can only move vertically. There are many short, combative Starchess games: openings tend to be more tactical than in Chess but endgames are of similar length.

After generating the sub-6-man (s6m) Starchess EGTs, Rusz identified 9,967,573 type B1-M zugs. As in Chess itself, B1-M zugs with $z u g$ depth $^{13} z d<3$ predominate: $9,852,307$ have $z d=1$ and many merely call for a waiting move; 78,001 have $z d=2 .{ }^{14}$ As in scenario 2, the question then arises as to which are vital type B1 zugs. A motivation is that if White can be forced to visit the btm side of the B1 zug in $t$ (transit

12 The hexagons are numbered in columns, left to right, and bottom to top: ' 37 ' is on the right.

${ }^{13}$ The zug-depth $z d$ of a B1-M zug is the difference between the wtm and btm DTM depths.

${ }^{14}$ Of 1,626,168,997 s6m positions, $0.61 \%$ are B1-M zugs: $0.0023 \%$ also have $z d>2$. 
length) moves, it is likely that a study-like scenario will be found. The technique, as in Section 3, is to see if, with the btm side of the B1 zug drawn, the B1 zug itself becomes a draw. If and only if it does, Black can force White's win to visit the btm position. The forced transits, wtm to btm position, may have different lengths.

Two observations speeded the identification of the vital s6m B1-M zugs, the first by Rusz and Starchess-specific. Vital B1 zugs in pawnful positions must have $z d>2$ : both sides must play at least two moves in going from the wtm to the btm position as there are no moves which preserve symmetry across the single vertical axis of symmetry. This meant that 7,168,489 B1-M zugs could be ignored, a splendid economy. Secondly, as in any game, if the B1-M zug in set TP is seen to be a win despite the btm position being defined as drawn, the generation of the Starchess(SP) EGT may be discontinued. Thus, over $50 \mathrm{~B} 1-\mathrm{M}$ zugs were examined in the time taken to generate an EGT. Fourteen of the 910 VB1 zugs found (30 4-man, 128 5-man pawnless) and one instructive non-VB1 zug, SC5, feature in Figures 7-8 and here.

KPPKP VB1 zug $S C 1$ is analogous to Lasker's chess study ${ }^{15}$ HHDBIV\#14482 [1, 23-25]. The max $t l$ line 1. K31"' (1. K20? K32"' 2. K21' K25"' 3. K16' K20' 4. K9' K14"' $z=)$ 1. ... K27' 2. K24"(') K34" 3. K19"(') K33" 4. K25"' z pl K34" 5. K20"(1) K27" 6. $\mathrm{P} 33+"{ }^{(1)} \mathrm{K} 33^{\prime} 7$ 7. K21"' K32' 8. K16"' K26" 9. K9"' K21" 10. K10 K20' 11. K16"(1) 1-0. A

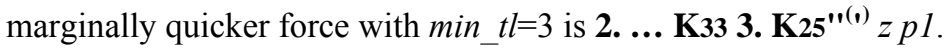

KQKB VB1 zug SC2 illustrates an important Q-triangulation. 1. Q20+"' K3" 2. Q21+"(') $\mathrm{K}^{\circ}$ 3. Q15"(') $z$ pl 1-0.

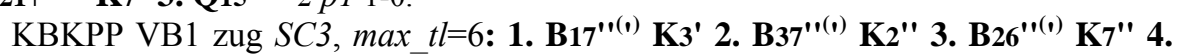
B33" K8" 5. B27+"(') K7 (-4) 6. B1"(') $z$ pl. Black may also play the min $t l=4$ line 2. ... K8 (-3) 3. B21+" ${ }^{\prime(1)} \mathrm{K} 7$ (-3) 4. B1"'(() illustrating the 'B-diamond' manoeuvre.

KNKRP VB1 zug $S C 4, t l=3$, three VB1 zugs: vzl 1. K9"(') K33' 2. K16"(') K27' 3.

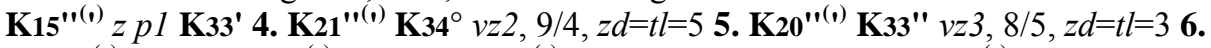

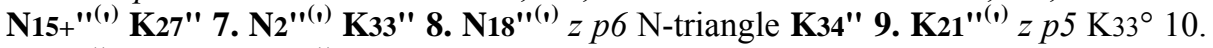
$\mathrm{N} 15+^{\prime(1)} \mathrm{K} 34^{\circ} 11 . \mathrm{K} 26^{\prime\left({ }^{(1)}\right.} \mathrm{P} 18^{\circ} 12 . \mathrm{N} 33^{\prime \prime \prime} \mathrm{P} 17=(\mathrm{Q} / \mathrm{R} / \mathrm{B} / \mathrm{N})^{\circ} 13 . \mathrm{N} 16 \#^{\prime \prime \prime} 1-0$.

KQKR position SC5 just fails to be a Vital B1 zug. The wK triangulates from/to the zug: 1. K33" K24" 2. K27" K31' 3. K26" z pl. However, setting $p 3 b$ to draw neatly reveals the dual win from $p 1 w$ (annotation in $\operatorname{Chess}(S P)$ terms): 1. K21"(') R28" 2. K15"(') distant wK/bR opposition! 2. ... R29" 3. K20' R30" 4. K14" ${ }^{\left({ }^{(}\right)}$R28" 5. K19"(')

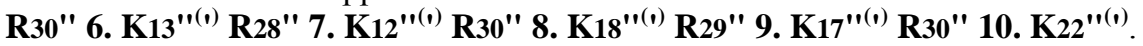

KBPKN VB1 zug $S C 6, t l=3: v z 1$ 1. B34"" K16" $v z 2,15 / 12, z d=3$ 2. B8"'(() K9" 3.B1"(1) $z$ pl (3.P3? K16"' z) 3. ... K16" 4. B34"(1) K9' 5. P3"(1) $z$ K16" 6. B8"' z K9" 7. $\mathrm{K} 25^{\prime \prime(1)} z \mathrm{~K} 16^{\prime \prime} 8 . \mathrm{K}_{2} 6^{\prime \prime(1)} z \mathrm{~K} 9 "$ 9. K21"(1) $1-0$.

KNPKN VB1 zug $S C 7, t l=3$ : one of three positions with a record $z d=25$. 1. K19'" N3" 2. K14"(') N10 (-22) 3. K13"'(') z pl. If 2. ... K18" then 3. N20"' N19" 4. P10N"" reaches an interesting KNNKN endgame, a general win.

KQRKB VB1 zug $S C 8$, $\max t l=12$, the second-longest known transit: 1. Q26+"()

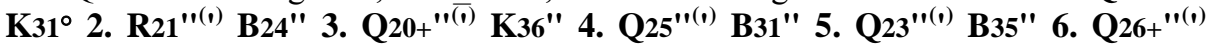
K31 ${ }^{\circ}$ 7. Q32+"(') K24 8. Q19+"(() K23" 9. Q17+"' K24" 10. Q18+"(!) K25 (-6) 11. Q36"(') B30" 12. R20"(') $z$ pl 1-0. A min_tl=7 line diverges 2. ... B35 (-4) 3.Q32+"(() K24 ${ }^{\circ}$ 4.Q19+"'(') K31 (-1) 5.Q18+"'(') K25 (-6) 6.Q36"'(') B30" 7.R20"'(') z p1.

${ }^{15}$ wKc5,Pa5,c6/bKc7,Pa6: 1. Kd5"' Kc8" 2. Kd4' Kd8' 3. Kc4"(1) Kc7 (-1) 4. Kc5"(1) z p1. 


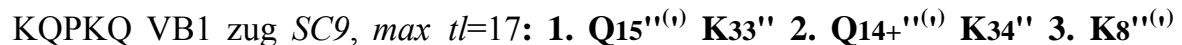

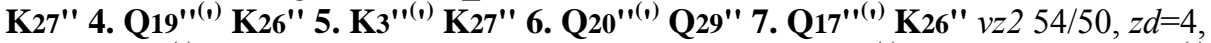
$t l=4$ 8. K2"(') Q34" 9. Q19'" Q28+" 10. K3'" Q29" 11. Q17"(() $z$ p 8 K27" 12. K8'(') Q31" 13. K14"(') Q24+" 14. K15"" Q25+' 15. Q20"(() Q13+ (-1) the only suboptimal

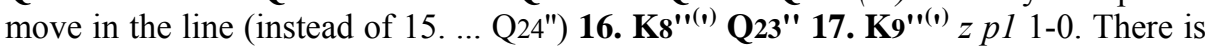
a $\min \_t l=3$ line diverging 2. ... K27 (-15) 3.Q20"'(') z pl 1-0: $\max \_t l-m i n \_t l=14$.
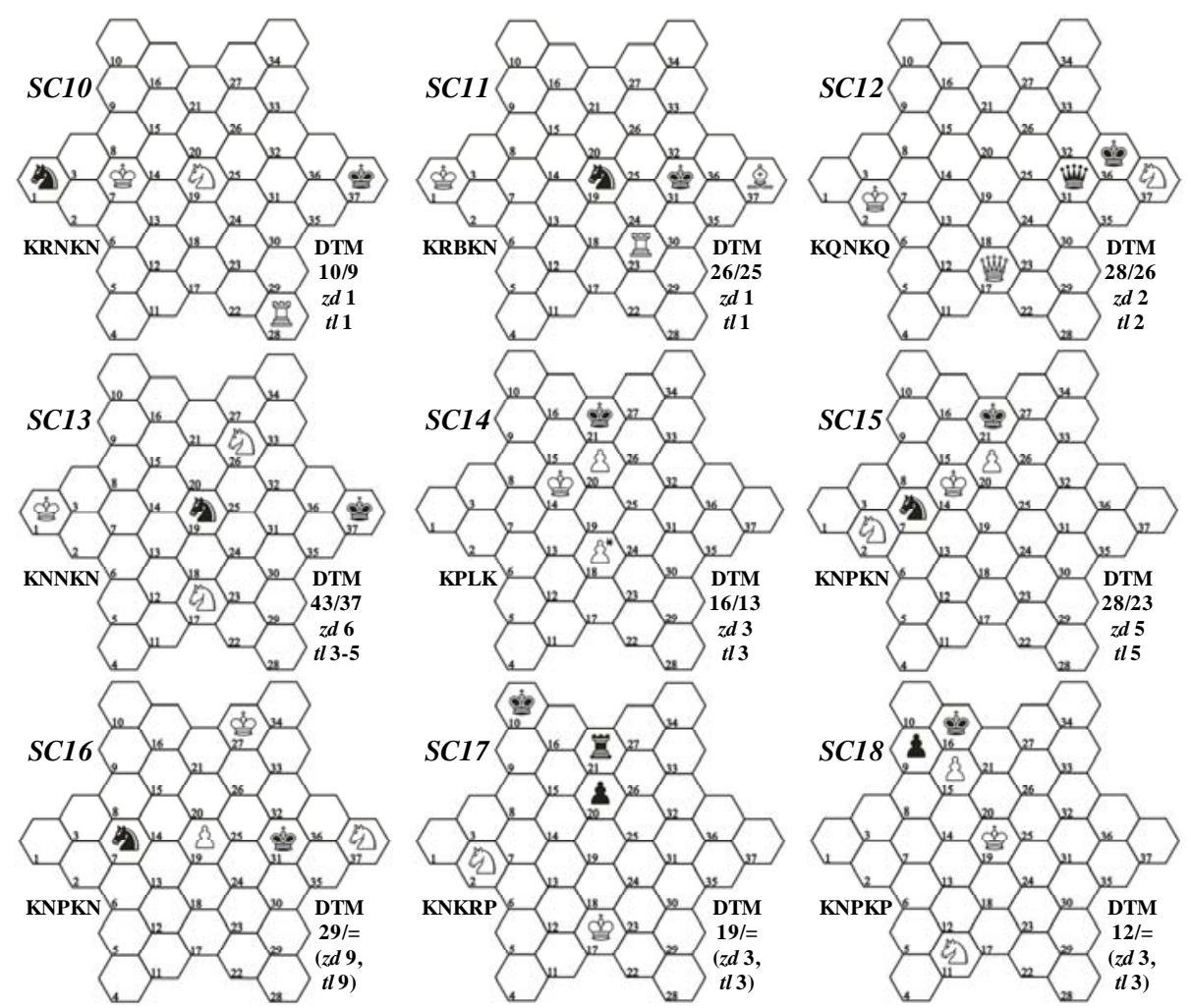

Figure 8. The six s6m reflected VB1 zugs and three studies featuring VB1 zugs.

The VB1 zugs of Figure 8 are the six s6m reflected ones: SC10-13 are reflected in the horizontal axis and $S C 14-15$ in the vertical axis. SC10-11 have $z d=1$ and $S C 12$ uniquely has $z d=2$. From $S C 13$, Black has the choice to reflect the vital zug or not. SC14 is the only reflected VB1 zug featuring a Limping Pawn, a pawn which appears to be able to move two squares but in fact cannot as it has already captured a man. The opening position and the dance of the Knights makes $S C 15$ visually remarkable.

KRNKN VB1 zug SC10, $t$ l=1: 1. R34'" z pl-reflected (denoted $p 1-r$ ) 1-0.

KRBKN VB1 zug $S C 11, t l=1$ : 1. R26"' z pl-r 1-0.

KQNKQ VB1 zug $S C 12, t l=2: \mathbf{1}$. Q21+" ${ }^{\left({ }^{(}\right)} \mathbf{K 3 5 " ~ 2 . ~ K 3 " ( { } ^ { ( 1 ) }}$ z pl-r 1-0.

KNNKN VB1 zug SC13, max $t l=5$ : 1. N31"(!) N35 (-1) 2. N26-13"(() N19" 3.

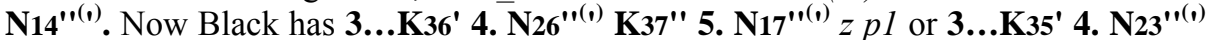
K37" 5. N21"(') z pl-r. min_tl=3: 1. ... K36 (-3) 2.N14"(') K37" 3.N17"(() z pl 1-0. 
KPLK VB1 $S C 14, t=3$ : 1. K19'" K27" 2. K24'(') K21" 3. K25'" z pl-r 1-0. White has the option, with the same tempo, not to reflect this VB1 zug. If the Limping Pawn were not limping, the position would be a non-vital type B1 zug (DTM 14/13): 1. K19" K16' 2. P21=Q' K21 z 3. K14' K26 z 4. K15"(1) K25 5. P20"(1) 1-0.

KNPKN VB1 SC15, $t=5$ : 1. N9"' N17" 2. K19"' N31" 3. N26"(') N27' 4. K25'" N31" 5. N35'"(') z pl-r 1-0.

The identification of the $910 \mathrm{~s} 6 \mathrm{~m}$ Vital B1 zugs has revealed about 250 study scenarios and many positions of pedagogic value. Here are three of them.

SC16, with a positional draw in line A and Knight sacrifices in both lines: 1. P20"'

a) 1...K30 2. N25'"! (2. K26? K23"' 3. N25 N24"' 4. K27 K22"' 5. N37 N7"' 6. K26

K23"' 7. N25 N24"' positional draw) 2. ...N24 3. N16" K23" 4. N19" K18 5. N11'!, or

b) 1...K32" $v z z d=t l=9$ 2. N29" N24" 3. N19"(') K25" 4. N36"(() N7"' 5. N34"' K32" 6. N16"'! N24" 7. N3"(() K25' 8. N12"' K32" 9. N29"(() N7" 10. N37"(()! p2 K31" 11. K26"(') K30" 12. N33"(') K23" 13. N19'(') K17" Black defends against the N11! sacrifice 14. N29'" N24 15. N37'! N7 16. N25" 1-0.

SC17: 1. N18'"' (1.N19?) 1...P19' 2. K23'"' K16' 3. K30'" R20' 4. K31'" K21 5. K25'"

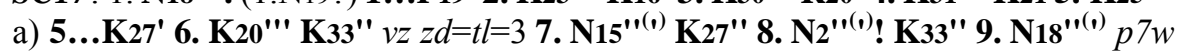

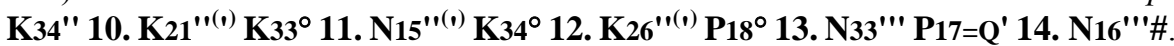

b) 5...K16' 6. K20"(') K9" $v z-r z d=t l=3$ 7. N26+"(') K16" 8. N35"(')! K9" 9. N18'(() $p 7 w$ K10" 10. K21"(') K9 ${ }^{\circ}$ 11. N26"(') K10 ${ }^{\circ}$ 12. K15"(() P18 $^{\circ}$ 13. N9"" P=17Q' 14. N27'"\#. Two echo variations with N-triangulation.

SC18: 1. N7+"' (1. K20? P15"'=) 1...K15" $v z z d=t l=3$ 2. N24"' K8" 3. N11"(')! K15" 4. $\mathrm{N7}^{\prime \prime()} p 2 \mathrm{~K}^{\prime \prime}$ 5. $\mathbf{N 2 4}{ }^{\prime\left({ }^{(}\right)} \mathrm{K} 3^{\circ}$ 6. N11"(')! an interesting, original N-manoeuvre K8'

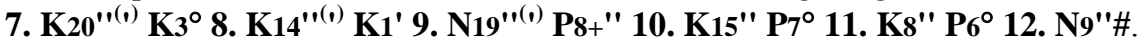

\section{Summary}

There is a clear need to identify all $\mathrm{s} 7 \mathrm{~m}$ time-wasting moves in the mainlines of studies in HHDBIV. This is a precursor to refining the study community's artistic judgement of that corpus [26] and would add considerable value to both. Proof statements should be derived by algorithm, reliable, economical, irredundant, comprehensible and verifiable: this is not the case at present. The approach using Chess $(S P)$ EGTs in principle yields proofs of moves' time-wasting status meeting the requirements stated. Further, it provides a tool to identify time-wasting moves in the future. This paper implicitly challenges the authors of EGT generator software to generalize that software to include variant games where some of White's won positions are deemed to be draws.

Similar questions of interest to endgame theoreticians, including those concerning lines from the deepest positions, zugzwang effects, 'downstream convergence' and Draw Studies, may be addressed using the same Chess(SP) approach. Other sources of endgame play, e.g., [19] [20], and studies' sidelines are worth inspection.

The authors particularly thank CESC [27], Rafael Andrist, John Beasley, Ian Bland, Eiko Bleicher, Marc Bourzutschky, Noam Elkies, Harold van der Heijden, Harm Müller, John Nunn, John Roycroft, John Tamplin and the three anonymous referees for their interest in and contributions to this paper. 


\section{References}

1. van der Heijden, H.M.J.F.: HHdbIV, HvdH's Endgame Study Database IV, http://www.hhdbiv.nl/ (2010)

2. Haworth, G.M ${ }^{\mathrm{c}}$ C., Bleicher, E., van der Heijden, H.M.J.F.: Uniqueness in chess studies. ICGA Journal, 34-1, 22-24 (2011)

3. Nalimov, E.V., Haworth, G.M ${ }^{\mathrm{c} C}$., Heinz, E.A.: Space-Efficient Indexing of Endgame Databases for Chess. Advances in Computer Games 9, (eds. H.J. van den Herik and B. Monien), pp. 93-113. Institute for Knowledge and Agent Technology (IKAT), Maastricht, The Netherlands. ISBN 9-0621-6576-1 (2001)

4. Bleicher, E.: DTM EGT query facility. http://www.k4it.de/index.php?topic=egtb\&lang=en (2007)

5. Pritchard, D.B.: Popular Chess Variants. Batsford. ISBN 978-0713485783 (2000)

6. Pritchard, D.B.: The Classified Encyclopedia of Chess Variants. 2nd revised edition edited and published by J.D.Beasley.

7. Karrer, P.: KQQKQP and KQPKQP $\approx$. ICCA Journal, 23-2, 75-84 (2000)

8. Bourzutschky, M., Konoval, Y.: News in Endgame Databases. EG 17-185, 220-229 (2011)

9. Konoval, Y., Bourzutschky, M.: Malyutka requiring $\mathrm{P}=\mathrm{R}$ and $\mathrm{P}=\mathrm{N}$. 64 Shakhmatnoe Obozrenie 7 (2009)

10.Konoval, Y., Bourzutschky, M.: First 6-man study requiring all three underpromotions. British Chess Magazine, 130-1 (2010)

11.Bourzutschky, M.S., Tamplin, J.A., Haworth, G.M ${ }^{\mathrm{c}}$ C.: Chess endgames: 6-man data and strategy. Theoretical Computer Science, 349-2, 140-157. ISSN 0304-3975 (2005)

12.Haworth, G.M ${ }^{\mathrm{c}}$., van der Heijden, H.M.J.F, Bleicher, E.: Zugzwangs in Chess Studies. ICGA Journal, 34-2, 82-88 (2011)

13.Grondijs, H.: No Rook Unturned. A Tour around the Saavedra Study (2004)

14.Rusz, A.: A technical discussion of the Hornecker study HHdbIV\#75649. http://kirillkryukov.com/chess/discussion-board-/viewtopic.php?f=6\&t=6036\#p64616 (2011)

15.Beasley, J.: Depth and Beauty, the chess endgame studies of Artur Mandler (2003)

16.Bleicher, E., Haworth, G.McC.: 6-man Chess and Zugzwangs. Advances in Computer Games (ed. H. Jaap van den Herik and P. Spronck), pp. 123-135. Proceedings of ACG12, Pamplona, 2009. Lecture Notes in Computer Science, 6048. Springer-Verlag (2010)

17.FIDE: The Laws of Chess. FIDE Handbook E.1.01A. http://www.fide.com/component/handbook/?id=124\&view=article (2009)

18.Haworth, G.M ${ }^{\mathrm{C}}$ C.: Strategies for Constrained Optimisation. ICGA J. 23-1, 9--20 (2000)

19.Nunn, J.: Secrets of Minor-Piece Endings. B.T. Batsford, London. (1995)

20.Nunn, J.: Secrets of Pawnless Endings. B.T. Batsford, London. ISBN 0-7134-7508-0. Expanded Edition 2 including 6-man endgames (2002). Gambit. ISBN 1-9019-8365-X (1994)

21.Vlasak, E.: Endgame database news. http://www.vlasak.biz/tablebase.htm (2005)

22.Starchess rules. polgarstarchess.com/Rules.doc (2011)

23.Winter, E.: Chess Notes \#5814. http://www.chesshistory.com/winter/winter51.html (2011)

24.Lasker, E.: Common Sense in Chess (1896)

25.Fahrni, H.: Das Endspiel im Schach. Leipzig (1917)

26.Roycroft, A.J.: Test tube chess: a comprehensive introduction to the endgame study (1972)

27.Haworth, G. $\mathrm{M}^{\mathrm{c}} \mathrm{C} .:$ The Scorched Earth Algorithm. Presentation to the UK Chess Endgame Study Circle. Pushkin House, London (2009)

28.Roycroft, A.J.: Expert against the Oracle. Machine Intelligence 11 (eds. J.E. Hayes, D. Michie and J. Richards), 347-373 (1988) 


\section{Appendix: further details of some positions in Table 1}

UP1, Karrer [7]. wKg4,Qg8,h8/bKf1,Qb4,Pd2 wtm:

This position is DTM=60 but a mate in 20 if Pawn-promotion is to Queen only.

1. Kf5"' Qc5+" 2. Ke4"' Qe7+" 3. Kf4" Qb4+' [Qc7+, Qd6+]

4. Ke3" Qc5+' [Qb6+, Qe7+] as d1=N is not available 5. Kxd2" $\{\mathrm{DTM}=15\}$

With the $\mathrm{P}=\mathrm{N}$ option, a DTM-minimaxing line starts:

1. Kf5"' Qc5+" 2. Ke4"' Qe7+" 3. Kf4" Qb4+' 4. Ke3" d1=N+" $\{K Q Q K Q N\}$

5. Kf3"' Qa3+" 6. Kf4"' Qe3+" 7. Kf5"' Qd3+' 8. Ke5" Qc3+" 9. Kd5" Qa5+"

10. Ke6' Qb6+" 11. Ke5' Qc7+" 12. Ke4" Qc2+".

UP2, Konoval and Bourzutschky [8-9], wKc8,Qf4,Pg7/bKh5,Qh1 wtm, requiring Pawn-conversion in different lines to respectively Queen, Rook and Knight:

1. Kc7"' Qh3'

(1.... Qg1 2. Qf7+"' Kh6' 3. g8=N+"' Kg5" 4. Qg7+" Kf4' 5. Qxg1"')

(1.... (Qa8/Qd5)" 2. Qh2+"' Kg6 3. Qg2+"' Qxg2" 4. g8=Q+"')

2. Qe5"' Kh6 3. g8=R"'.

UP3, Konoval and Bourzutschky [8], [10]. wKc2,Rb5,d5,Pg5/bKh5,Qf8 wtm, the first s7m study synthesising all three Rook, Bishop and Knight underpromotions:

1. g6"' Kh6" 2. g7"' Qf2+" 3. Rd2"' (DTM = 34) and now

3. ... Qf3 4. g8=R"' Qc6+" 5. Kd1"' Qh1" 6. Ke2" (6. Kc2 Qc6" 7. Kd1"' Qh1"),

3. ... Qf4" 4. g8=B"', or

3. ... Qf1 4. g8=N"' Kh7' 5. Rb7+"' Kh8" 6. Ne7"'.

$P H$, the Philidor position (1777). wKc6,Qa5/bKb8, Rb7 wtm/btm, a B1 zug:

btm: 1. ... Rb1" 2. Qd8+' Ka7 3. Qd4+" (Ka8/b8)" 4. Qh8+" Ka7" 5. Qh7+".

wtm: 1. Qe5+' Ka8' 2. Qa1+' Kb8" 3. Qa5" arriving at the btm line above.

$\mathrm{KH}$, the Kling and Horwitz position (1851). wKd5,Ba4,f8/bKb6, $\mathrm{Nb} 7 \mathrm{wtm}$.

This position was long thought to be drawn: in fact, DTC $=45 \mathrm{~m}$ and DTM $=57 \mathrm{~m}$.

White has to force Black from the pseudo-fortress [28] and prevent a similar pseudofortress being set up. This DTC-minimaxing line shows the difficulty involved.

1. Bb4" Kc7' 2. Bd2' Kb6" 3. Be3+' Kc7" 4. Bf2' Nd8' 5. Kc4' Nb7"

6. Bg3+' Kb6" 7. Kb4" Nd8" 8. Bf2+" Kc7" 9. Kb5" Ne6" 10. Bg3+' Kd7"

11. Bd1" Nd4+" 12. Kc5" Nf5" 13. Be5' Kd6" 14. Bc3" Ne3" 15. Bf3" Kf5"

16. Bc6" Nf1' 17. Kd5" Ng3" 18. Bd7+' Kf4" 19. Bd2+' Kf3 ${ }^{\circ}$ 20. Bh6' Nf1'

21. Kd4" Ng3' 22. Bc6+' Kg4" 23. Ke5" Ne2' 24. Be3' Ng3' 25. Bc5' Nh5'

26. Bb6' Ng3" 27. Ba4" Nf1' 28. Bd1+" Kg3" 29. Kd4' Kf2' 30. Kd3+" Kg3'

31. Ke2" Nh2" 32. Bc7+" Kh3" 33. Ba4' Ng4" 34. Bc6" Kh4" 35. Kf3" Kh5"

36. Kf4" Nf6" 37. Kf5" Ng8" 38. Bf3+" Kh6" 39. Bd6" Kg7" 40. Kg5" Kf7"

41. Bd5+" Kg7" 42. Bc4' Kh7 43. Bf8" Kh8" 44. Bd3" Ne7' 45. Bxe7"

$B 1 Z$, Elkies' pedagogic example (2011). wKg5,Pe6,f7/bKg7,Pe7,g6 wtm.

The wK cannot capture bPe7 first: with the bK on $\mathrm{f} 8$, the g-Pawn just advances.

Therefore the triangulation is necessary and the win must visit $B 1 Z$ with btm.

1. Kf4' Kf8" 2. Kg4" Kg7" 3. Kg5"(') B1Z-btm Kf8" 4. Kh6"(') g5 ${ }^{\circ}$ 5. Kxg5"

Kg7 ${ }^{\circ}$ the type B1 zug $B 1 Z^{\prime}$ : the win need not visit $B 1 Z^{\prime}$ with btm. 6. Kf5" Kf8". 